\title{
A New Concept for High-Elevation Radio Detection of Tau Neutrinos
}

\author{
Stephanie Wissel ${ }^{1, \star}$, Jaime Alvarez-Muñiz ${ }^{2}$, Washington R. Carvalho, Jr. ${ }^{3,2}$, Andrés Romero- \\ Wolf ${ }^{4}$, Harm Schoorlemmer ${ }^{5}$, and Enrique Zas ${ }^{2}$ \\ ${ }^{1}$ California Polytechnic State University, San Luis Obispo, CA, 93407, USA \\ ${ }^{2}$ Departamento de Física de Partículas \& Instituto Galego de Física de Altas Enerxías, Universidade de \\ Santiago de Compostela, 15782 Santiago de Compostela, Spain \\ ${ }^{3}$ Departamento de Física, Universidade de São Paulo, São Paulo, Brazil \\ ${ }^{4}$ Jet Propulsion Laboratory, California Institute of Technology, Pasadena, CA 91109, USA \\ ${ }^{5}$ Max-Planck-Institut für Kernphysik, 69117, Heidelberg, Germany
}

\begin{abstract}
Cosmic neutrinos are expected to include a significant flux of tau neutrinos due to flavor mixing over astronomical length scales. However, the tau-neutrino content of astrophysical neutrinos is poorly constrained and a significant flux of cosmogenic tau neutrinos awaits discovery. Earth-skimming tau neutrinos undergo charged-current interactions that result in a tau lepton exiting the Earth. The tau lepton decay generates an extensive air shower and geomagnetic radio emission. To target the tau neutrinos, we present a new tau neutrino detector concept that uses phased antenna arrays placed on high elevation mountains. Simulation studies indicate that a modest array size and small number of stations can achieve competitive sensitivity, provided the receivers are at high enough elevation.
\end{abstract}

Tau neutrinos are expected to be a significant component of the ultra-high-energy (UHE, $E>$ $10^{17} \mathrm{eV}$ ) cosmic neutrino flux. Earth-skimming tau neutrinos can generate tau leptons emerging from the Earth, after the tau neutrinos interact via a charged current interaction. A radio detector pointed at the horizon can search for the impulsive band-limited radio emission generated when the tau lepton decays. In this study, we consider the sensitivity of a new detector concept called the Beamforming Elevated Array for Cosmic Neutrinos (BEACON). The goal of BEACON is to search for tau neutrinos on top of high-elevation mountains with phased arrays.

The acceptance of BEACON depends on the geometric acceptance at different elevations [1], the probability that a $\tau$ will exit the Earth [2] and decay to produce an air shower before reaching the detector, and the probability that BEACON will trigger on the event. To estimate the probability that BEACON will trigger on a $\tau$-induced air shower, we generated a library of ZHAireS [3] pulses from upgoing $\tau$ 's decaying at ground level through the dominant decay mode $\tau \rightarrow \pi^{-} \pi^{0} v_{\tau}$. The radio emission is received at several detectors placed on a line perpendicular to the shower axis. The peak electric field at each detector is stored in look-up tables binned in 10-MHz subbands, $\tau$ emergence angles from the horizon at the exit point, view angles from the $\tau$ decay point, $\theta_{\text {view,decay }}$, and detector altitudes. We then interpolate the electric field in the lookup-tables, integrate over a selected frequency

^e-mail: swissel@calpoly.edu 

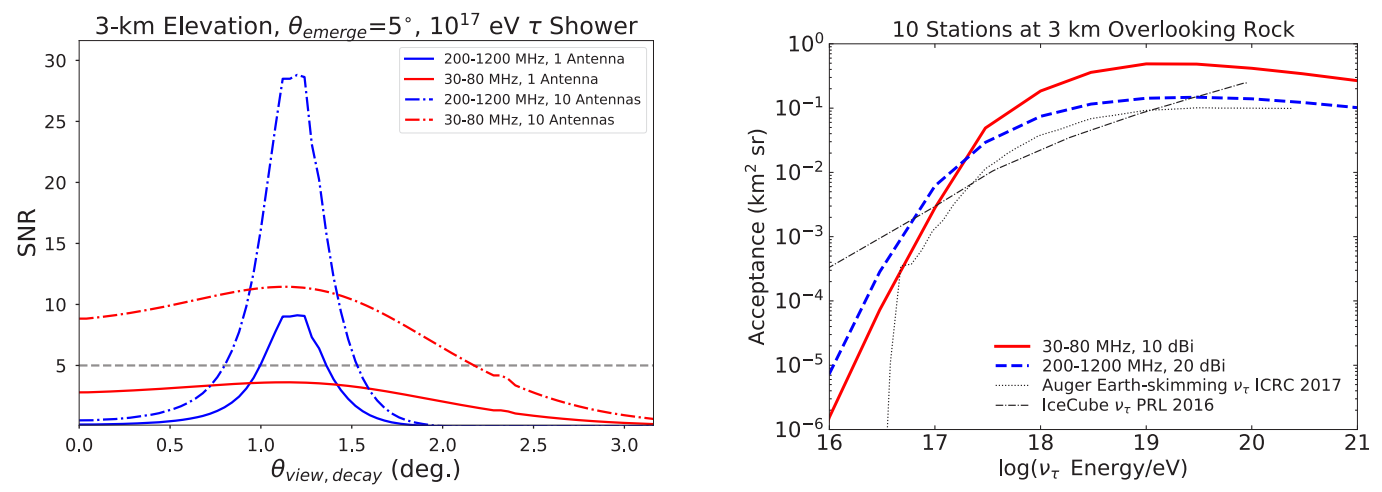

Figure 1. Left: $\mathrm{A} 10^{17} \mathrm{eV} \tau$ shower emerging at a $5^{\circ}$ and decaying at the exit point generates a radio beam pattern shown here as the SNR versus $\theta_{\text {view,decay }}$. The radio emission is filtered in the $30-80 \mathrm{MHz}$ (red) and $200-1200 \mathrm{MHz}$ bands (blue) for 1 antenna (solid) and 10 phased antennas (dot-dashed). Right: The acceptance of 10 mountaintop radio detectors to $v_{\tau}$ 's compared to IceCube's acceptance to $v_{\tau}$ 's [5] and the Pierre Auger Observatory's acceptance to Earth-skimming $v_{\tau}$ 's [6].

band, and scale by the energy and distance from the detector to the exponentially sampled decay point to calculate the signal-to-noise ratio (SNR) for each shower in a Monte Carlo acceptance calculation. The SNR is defined here as the peak voltage from the $\tau$ shower divided by the root-mean-square voltage from a frequency-dependent thermal noise model that includes galactic noise [4], thermal noise from the ground, and a system temperature of $140 \mathrm{~K}$. If the SNR is higher than 5.0, a simulated shower will trigger the detector. The radio beam pattern filtered in two different frequency bands from a tau emerging at $5^{\circ}$ as a function of the view angle from the tau decay point relative to the $\tau$ shower axis, $\theta_{\text {view, decay }}$, is shown on the left of Fig. (1). The lower frequency (VHF, 30-80 MHz) beam pattern is broader that the higher frequency design (UHF, 200-1200 MHz), but reaches a lower peak SNR.

Phased arrays increase the gain and acceptance of a single mountaintop station. In a phased array, multiple antennas are delayed and summed coherently to form beams. The beam SNR increases with the square-root of the number of antennas, $N$, (see Fig. (1) left), and the gain in decibels increases with $\log N$. Multiple beams can cover the full azimuthal field-of-view available at a given mountain site with higher gain than a single antenna and can be used to reject localized anthropogenic backgrounds.

Fig. (1) right compares two possible mountaintop experiments placed at $3 \mathrm{~km}$ elevation. Each experiment is composed of 10 stations. The VHF design uses 7 electrically short dipoles each with a gain of $1.8 \mathrm{dBi}$ that are phased together to achieve a gain of $10 \mathrm{dBi}$, while the UHF design uses 10 broadband directional antennas with individual gains of $10 \mathrm{dBi}$ and an effective gain of $20 \mathrm{dBi}$. At low energies $E_{\nu_{\tau}}<3 \times 10^{17} \mathrm{eV}$, the higher SNR of the UHF beams translate into a higher acceptance, but at higher energies, the broader beams of the VHF design result in a higher acceptance. These results indicate that a modest experiment size of 10 stations can improve on the acceptances to $10^{17}-10^{19} \mathrm{eV}$ tau neutrinos from IceCube [5] and the Pierre Auger Observatory's Earth-skimming channel [6].

Fig. (2) shows the number of neutrinos expected from cosmogenic models with mixed composition [7]. The event rate increases linearly with the number of antennas up to 10 antennas, but sub-linearly above that. This suggests that BEACON should phase at most 10 antennas. On the right, we assume station designs with 7 VHF (10 dBi) and $10 \mathrm{UHF}(20 \mathrm{dBi})$ antennas. The number of events from cosmogenic models increases nearly linearly with the elevation of the station up to $3 \mathrm{~km}$. However, 


\section{Mixed CR Composition, Kotera 2010}
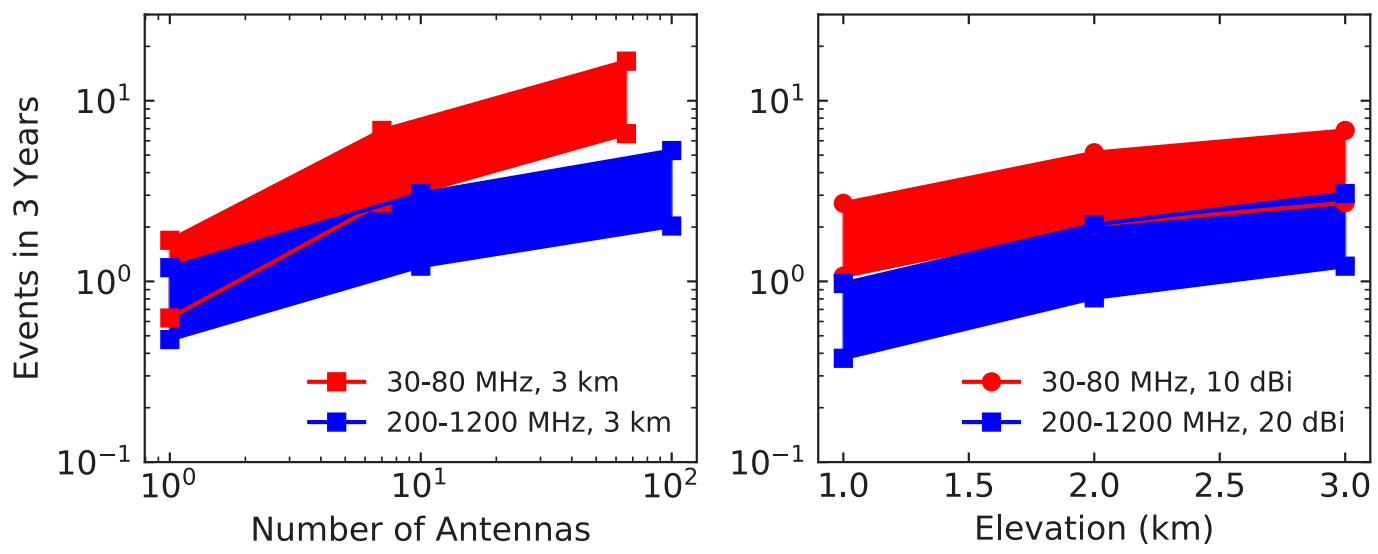

Figure 2. The number of events recorded in 3 years on 10 stations overlooking rock with a $120^{\circ}$ field-of-view, assuming the range of cosmogenic models with mixed cosmic ray composition from Ref. [7]. (Left) The detector elevation is fixed at $3 \mathrm{~km}$, but the number of antennas per station varies. (Right) The number of antennas is fixed at 7 for VHF and 10 for UHF, while varying the station elevation.

the number expected from extrapolations of the IceCube astrophysical fluxes [8, 9] or models with cutoffs $E<10^{18} \mathrm{eV}$ [10] are insensitive to elevation changes above $1 \mathrm{~km}$. At any elevation, the number of events received by the VHF design is a factor of two higher than with a UHF design.

The radio detection of $v_{\tau}$ 's from high elevations is a promising method for discovering UHE $v$ 's. We have calculated the acceptance and expected number of neutrinos to several detector designs using phased arrays. Ten mountaintop stations can test predictions from cosmogenic models with mixed cosmic ray composition. This optimization study can be used in the development of future experiments such as BEACON, TAROGE [11], and GRAND [12]. This work is supported in part by NSF Award 1752922.

\section{References}

[1] P. Motloch, N. Hollon, P. Privitera, Astropart. Phys. 54, 40 (2014)

[2] J. Alvarez-Muñiz et al., PRD 97, 023021 (2018)

[3] J. Alvarez-Muñiz, W.R. Carvalho, E. Zas, Astroparticle Physics 35, 325 (2012)

[4] G.A. Dulk, W.C. Erickson, R. Manning, J.L. Bougeret, A\&A 365, 294 (2001)

[5] The IceCube Collab., PRL 117, 241101 (2016)

[6] E. Zas, for the Pierre Auger Collab., Proc. ICRC 301, 972 (2017)

[7] K. Kotera, D. Allard, A. Olinto, JCAP p. 013 (2010)

[8] The IceCube Collab., ApJ 833, 3 (2016)

[9] The IceCube Collab., ApJ 809, 98 (2015)

[10] A. Romero-Wolf, M. Ave, accepted JCAP, arXiv:1712.07290 (2018)

[11] J.W. Nam et al., Int'1 J. of Mod. Phys. D 25, 1645013 (2016)

[12] K. Fang for the GRAND Collab., Proc. ICRC 301, 996 (2017) 